\title{
SCALING PATTERNS OF NATURAL URBAN PLACES AS A RULE FOR ENHANCING THEIR URBAN FUNCTIONALITY USING TRAJECTORY DATA
}

\author{
T. $\mathrm{Jia}^{1, *}, \mathrm{X} . \mathrm{Yu}^{1}$ \\ ${ }^{1}$ School of Remote Sensing and Information Engineering, Wuhan University, 430079 Wuhan, China - tao.jia@ whu.edu.cn
}

Commission III Urban Sensing and Mobility

KEY WORDS: Scaling patterns, Natural urban place, Urban functionality, Trajectory data

\begin{abstract}
:
With the availability of massive trajectory data, it is highly valuable to reveal their activity information for many domains such as understanding the functionality of urban regions. This article utilizes the scaling patterns of human activities to enhance functional distribution of natural urban places. Specifically, we proposed a temporal city clustering algorithm to aggregate the stopping locations into natural urban places, which are reported to follow remarkable power law distributions of sizes and obey a universal law of economy of scale on human interactions with urban infrastructure. Besides, we proposed a novel Bayesian inference model with damping factor to estimate the most likely POI type associated with a stopping location. Our results suggest that hot natural urban places could be effectively identified from their scaling patterns and their functionality can be very well enhanced. For instance, natural urban places containing airport or railway station can be highly stressed by accumulating the massive types of human activities.
\end{abstract}

\section{INTRODUCTION}

Human activities, such as shopping or working in the daily life, tend to be clustered in space and time. These hotspots or clusters are named as natural urban places in our work. This is because they are defined in a natural way using a bottom-up approach, namely individual activity locations (stopping locations) interact with each other using the same rule to group together as the final clusters ( $\mathrm{Jia}$ and $\mathrm{Ji}, 2017$ ). However, there are two points worthy of in-depth investigation owing to the insufficient studies in the literature. On the one hand, very few studies applied the scaling pattern of urban places to identify the ones whose functionality can be enhanced. On the other hand, to infer the urban functionality of urban places is not trivial in the literature (Furletti et al., 2013; Moiseeva et al., 2010; Zhang et al., 2015), and the studies of such kind might incorrectly estimate the urban functionality.

Therefore, this study is dedicated to investigate the scaling patterns of natural urban places and further apply this rule to the process of enhancing their urban functionality. First, we aggregate the stopping locations into natural urban places using a novel temporal city clustering algorithm (Please refer to Jia and Ji, 2017 for the detailed technique for extracting stopping locations). Second, the scaling patterns of natural urban places were examined, which is used to identify the hot natural urban places with a large number of stopping locations and a relative small number of POIs. Finally, a Bayesian inference model with damping factor was used to enhance the urban functionality of these hot natural urban places.

\section{SCALING PATTERN OF NATURAL URBAN PLACES}

\subsection{Deriving the natural urban places}

Natural urban places refer to the hotspots or clusters within urban area where most of the human activities are taken place, which are defined in a natural way using a bottom-up approach. We develop a novel temporal city clustering algorithm (TCCA), which is an extension of city clustering algorithm (CCA) (Rozenfeld et al., 2008) by considering the temporal dimension. It constructs the natural urban places by aggregating the individual stopping locations "from the bottom up".

Specifically, this method starts from a randomly selected location, and then a cylinder is drawn around it to check whether other locations are encompassed. The cylinder is constructed by drawing a circle with a specified spatial radius $r$, which is then extruded along the time dimension with a specified time resolution $\tau$. This process goes recursively until no locations are within the cylinder or the contained locations have already been checked. The novel aspect of TCCA is that it can be employed to handle massive location data in a distributed computing environment, which would be impossible with the conventional CCA. As shown in Figure 1, TCCA first divides the entire area into many groups according to time, and then assigns each group to a computation node for deriving clusters, and finally clusters from different computation nodes are merged or not depending on the temporal lag. In this study, we intuitively set the spatial radius $\mathrm{r}$ at $200 \mathrm{~m}$, which is the approximate average road length in the study area; we set the time resolution $\tau$ from $5 \mathrm{~min}$ to $30 \mathrm{~min}$ with 5 -min increments, which results in 6 groups of natural urban places. 


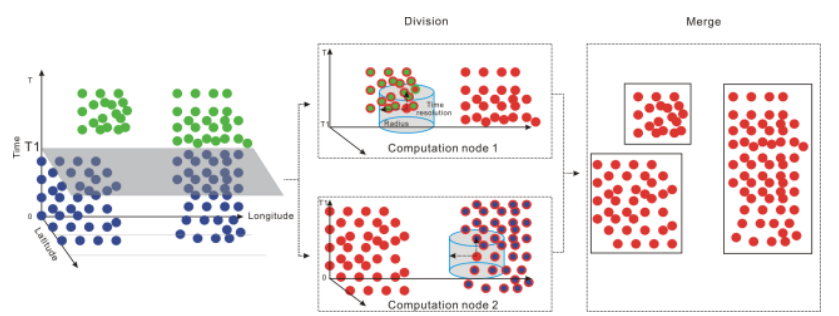

Figure 1. TCCA aggregating individual stopping locations.

\subsection{Scaling patterns of natural urban places}

We first examine the scaling patterns of natural urban places with respect to the number of stopping locations. The stops number might reflect regional prosperity, which can be approximated well by a power law distribution (Figure 2a), regardless of time resolution. This observation strengthens our understanding that natural urban places within a city closely resemble cities in city systems.

Next, we investigate the scaling relationship between the number of stopping locations and the number of POIs or road length. The number of POIs relates to the potential functionality of the urban place, while the length of roads denotes the accessibility of transportation for consuming services. The findings coincide with our initial assumption suggesting a clearly allometric relationship (Figure $2 \mathrm{~b}$ and $2 \mathrm{c}$ ), which implies that the universal law of economies of scale dominate human interaction with urban infrastructure. In addition, the allometric relationship remains stable regardless of clustering temporal resolution. Furthermore, the POI number is linearly correlated with road length (Figure $2 \mathrm{~d}$ ), which is statistically significant with a p-value of 0 by using F-test. This relationship remains stable and significant regardless of temporal resolution.

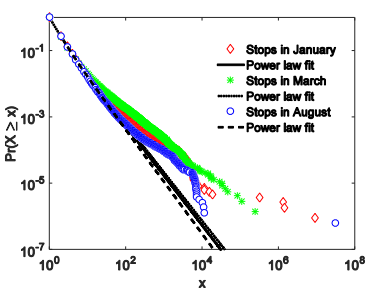

(a)

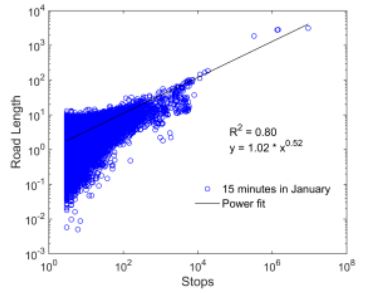

(c)

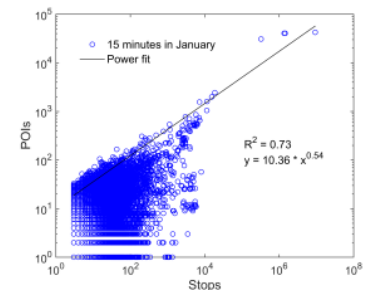

(b)

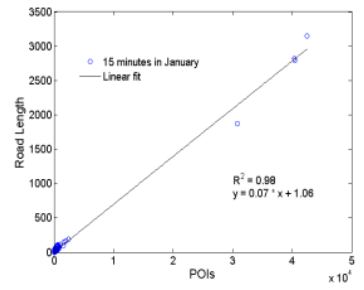

(d)
Figure 2. Scaling patterns of natural urban places

\section{FUNCTIONALITY ENHANCEMENT OF NATURAL URBAN PLACES}

\subsection{Selection of natural urban places for functionality enhancement}

It is our assumption that humans collectively interact with the urban infrastructure in an efficient way known as the economies of scale (Bettencourt et al. 2007), which means that we could roughly estimate the optimal required resource from the size of human activities. In fact, most of the natural urban places might be underestimated owing to the insufficient number of POIs compared with the number of stopping locations as shown in Figure 2c. These places might also have some very high popular POIs. As an example, we select a few such natural urban places that deviate significantly from the power fit line and have a large number of stopping locations. As shown in Figure 3, a total number of 66 natural urban places are selected and are spatially related to 6 places as shown in Figure 3.

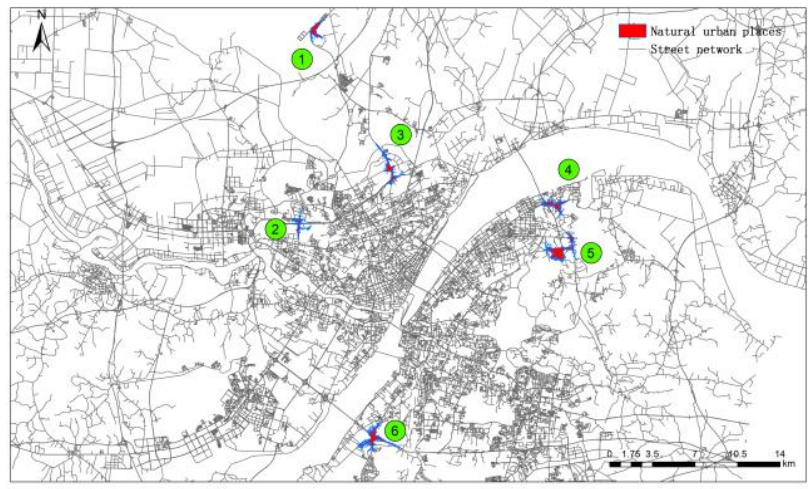

Figure 3. Selection of natural urban places for functionality enhancement.

\subsection{A Bayesian model to enhance the urban functionality}

The Bayesian inference model is constructed as follows. The first step is to derive the empirical probability of observing one stopping location around time $t$, given a POI type; the second step is to derive the empirical probability of observing one stopping location at coordinate $x, y$, given a POI type; and the third step is to infer the activity type of a stopping location with the maximum probability. To mimic the real urban functionality of natural urban places, we add the damping factor $\beta$ to the following inference model. In this context, it allows the stopping location with a probability to be matched with a POI type in the entire study area to supplement the urban functionality of a natural urban place.

$\operatorname{Pr}\left(t_{y p e} \mid(x, y), t\right)=\left\{\begin{array}{cl}\frac{\operatorname{Pr}((x, y) \mid t y p e) \bullet \operatorname{Pr}(t \mid t y p e) \bullet \operatorname{Pr}\left(t_{y p e}\right)}{\operatorname{Pr}((x, y), t)} & \beta \leq \beta_{0} \\ \frac{\operatorname{Pr}(t \mid t y p e) \bullet \operatorname{Pr}(t y p e)}{\operatorname{Pr}((x, y), t)} & \beta>\beta_{0}\end{array}\right.$

where $\quad t=$ the time of the stopping location

$x, y=$ the coordinates of the stopping location type $=$ the type of POI

$\beta=$ the damping factor

$\beta_{0}=$ the damping factor with a certain threshold

Once apply this model to the stopping locations of the selected natural urban places, we could obtain the corresponding enhanced urban functionalities. The results are shown in Figure 4 , where we can see clearly that the functionality of natural urban places are enhanced reliably. For instance, the natural urban place with ID 1 is a hot urban place by providing the aviation service, which is clearly enhanced by a significant number of stopping locations with the activity types of airport; the natural urban place with ID 5 is clearly enhanced by matching a huge number of stopping locations to the POI type of railway station, and hence it is considered as a railway service area, which agrees well the real land use pattern; the natural urban place with ID 6 is also clearly enhanced by emphasizing the importance of education, and hence, it is regarded as the place mainly providing education services, 
which is compatible with the three institutions or universities there by visual check on the on-line map service. The findings reported here suggests that the functionality of an urban place can be enhanced or accumulated by the types of massive human activities. With the availability of massive crowdsourced data in terms of human movement, it facilitates the automatic recognition of the functionality of urban places via a computer program without the interruption of human endeavour.
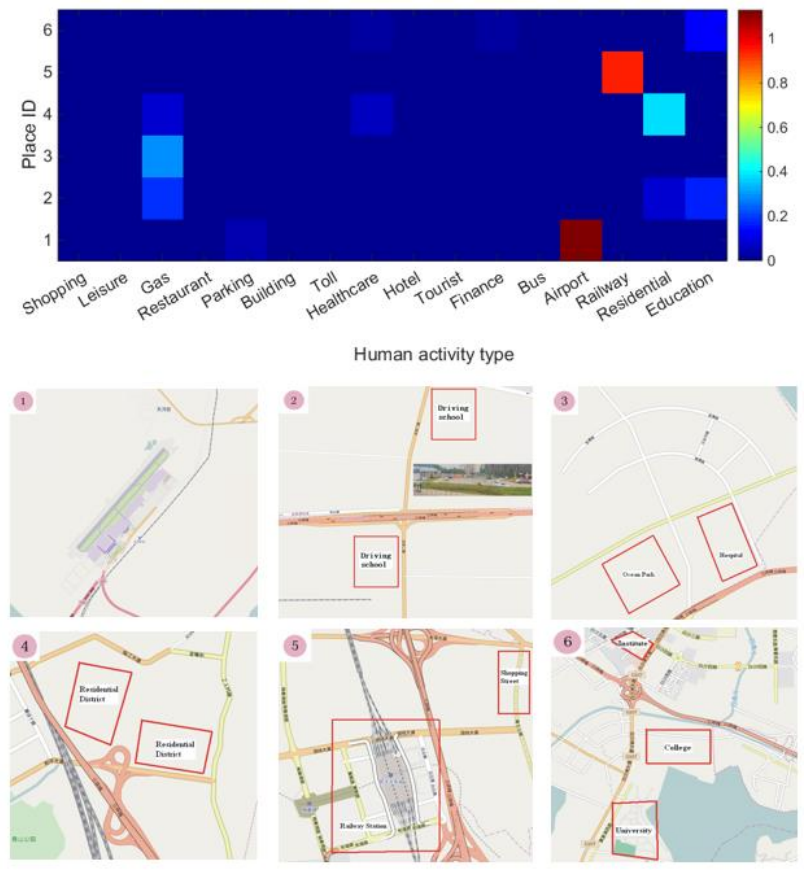

Figure 4. The enhanced urban functionality of the selected natural urban places.

\section{CONCLUSION}

Using massive taxicab GPS trajectory data, we proposed the method to derive natural urban places and to solve the problem of functionality enhancement of natural urban places. The scaling patterns of natural urban places give us the rule to identify the hot natural urban places whose urban functionality could be enhanced by accumulating the massive human activity types. Our results suggest that most of the hot natural urban places could be enhanced in terms of functionality distribution. The results would be useful in many urban applications, such as urban planning.

\section{ACKNOWLEDGEMENTS}

Thanks for the financial support from National Natural Foundation of China with the NO. 41401453.

\section{REFERENCES}

Jia, T., Ji, Z., 2017. Understanding the Functionality of Human Activity Hotspots from Their Scaling Pattern Using Trajectory Data. ISPRS International Journal of Geo-Information, 6(341), pp.1-16.

Furletti, B., Cintia, P., Renso, C., Spinsanti, L., 2013. Inferring human activities from gps tracks. In: Proceedings of the 2 nd ACM SIGKDD International Workshop on Urban Computing, Chicago, IL, USA, 11 August 2013.
Moiseeva, A., Jessurun, J., Timmermans, H., 2010 Semiautomatic imputation of activity-travel diaries using GPS traces, prompted recall, and context-sensitive learning algorithms. Transportation Research Record Journal of the Transportation Research Board, 2183, pp.60-68.

Zhang, W., Qi, G., Pan, G., Lu, H., Li, S., Wu, Z., 2015. CityScale social event detection and evaluation with taxi traces. ACM Transactions on Intelligent Systems and Technology, 6(3), 40.

Rozenfeld, H.D., Rybski, D., Andrade, J.S.Jr., Batty, M., Stanley, H.E., Makse, H.A., 2008. Laws of population growth. Proceedings of the National Academy of Sciences of the United States of America, 105, pp.18702-18707. 